American Journal of Pharmaceutical Education 2016; 80 (6) Article 92.

\title{
VIEWPOINT
}

\section{The Tweet Heard Round the (Health Professions) World}

\author{
Editorial Team, American Journal of Pharmaceutical Education
}

The title may obviously be tongue and cheek (and of course a tweet would be retweeted, not heard), but the intention behind it is sincere. The American Journal of Pharmaceutical Education (AJPE) recently launched a social media presence with the goal of expanding our ability to disseminate the valuable research published in the journal. Social media takes full advantage of the speed and breadth of digital communication, which is critical in a time when increasing visibility is key to the longevity of content. After all, the more people are aware of the work our authors do, the more people will associate AJPE with the leading edge of pharmacy education research and innovation.

Reaching a larger audience should be a goal of all health professions as interprofessional, patient-centered care becomes more prevalent. By having a social media presence, the AJPE editorial team is taking a major step in this direction. But we can't achieve this goal alone. As readers of the journal, but more importantly, as educators, researchers, authors, and health care providers, your help is invaluable. Follow us on Twitter, yes, of course-@TheAJPE. But please also share our posts. Retweet, and encourage colleagues and students - in pharmacy, higher education, and in other health professions - to join AJPE's Twitter family and share the work. Let us know who we should be following and whose work we should be sharing. Comment, participate, and join the global discussion.

In the end, the more information the higher education and health care world can share, discuss and improve upon, the better educated health care teams will be. And that translates to the best outcome of all: better cared for patients. 